ESAIM: PS 16 (2012) Sp-1

DOI: $10.1051 / \mathrm{ps} / 2012023$
ESAIM: Probability and Statistics www.esaim-ps.org

\title{
Editorial
}

\section{Spring School Mons Random differential equations and Gaussian fields}

This special issue of ESAIM P\&S originates from the spring school held in Mons, France, 15-19 June 2009. This spring school "Random differential equations and Gaussian fields" was supported by the French "Agence Nationale de la Recherche". It was organized around four main lectures given by Fabrice Baudoin (Purdue) "Stochastic Taylor expansions and heat kernels asymptotics", Laure Coutin (Toulouse) "Introduction to rough paths theory with applications", Serge Cohen (Toulouse) and Michael Lifshits (St Petersburg) "Stationary Gaussian Random fields on Hyperbolic Spaces and Euclidean Spheres" and Jacques Istas (Grenoble) "Manifold indexed fractional fields". This issue is constituted of the four lectures notes. 